\title{
Studies of High-accuracy Stress Calculation Method in Isogeometric Structure Analysis
}

\author{
He Gang, Gu Hao, Pan Zhengyu \\ College of Mechanical \& Electrical Engineering \\ Hohai University \\ Changzhou, China \\ ihegang@163.com
}

\begin{abstract}
The stress calculated method of isogeometric structure analysis is studied in this paper. Based on the fact that stress calculated values at Guass integral points are more accurate than other locations, the stress field can be rebuilt by fitting the Guass integral point's stress value from the displacement field, and two methods including stress interpolation and least square fit are presented to reduce the stress error. The example of infinite plate with circular hole is used to illustrate our methods' performance, and the results show that our methods can improve the stress calculate accuracy notable.
\end{abstract}

Keywords- isogeometric analysis, linear elastic, stress error, stress interpolation, least square method component

\section{INTRODUCTION}

Isogeometric analysis introduced by Hughes ${ }^{[1]}$, is using the basis functions to describe both the geometry and physics fields during analysis process. Since the geometry and the design boundary are usually represented by the parameter surfaces and curves such as NURBS and T-spline exactly, it is possible to have a more accurate solution than the conventional finite element method with fewer degrees of freedom, and an additional design parameterization is unnecessary. The analysis is running without the help of mesh regeneration. so the time-consuming meshing process is also saved. With these advantages, isogeometric analysis based on NURBS and T-spline ${ }^{[1}$ has now been widely researched in many different fields, such as structure design and optimization ${ }^{[2,3]}$, vibration ${ }^{[4]}$, fluid ${ }^{[5]}$ and contacted problem $^{[6]}$. It is proved that isogeometric analysis have great potential in many applications, espeically in shape optimization, fluid-structure interaction and CAD and CAE integration $^{[7]}$.

Linear elastic analysis is the earliest application aspects of isogeometric analysis. Analysis precision is a critical aspect of numerical method ${ }^{[8]}$. Although isogeometric analysis is running on a accurate geometric model, numeriacal error is unavoidable because of numerical integral and partial differential equation's discretization ${ }^{[1]}$. The accuracy is increased with the size of elements gradually. However, when the geometry is complex, the consumption on time and memory is huge. Linear elastic analysis is often built on displacement, but in fact stress is a more important response than displacement, and the error of stress is usually larger than that of displacement because stress is a higher order differential than displacement ${ }^{[8]}$.
In order to reduce the stress's error in linear elastic isogeometric analysis, two improved methods including stress interpolation and least square fit method are presented, which take advantage of the fact that the numerical points generally get more accurate stress calculated results. Two examples are given by comparing the stress results gotten by our methods with their theory solution.

\section{ISOGEOMETRIC ANALYSIS BASED ON NURBS}

\section{A. NURBS surface}

NURBS is the extension of B-spline, which has the ability to efficiently represent complex freeform surface and conic surface exactly. NURBS is the weighted and rational form of B-spline.NURBS surface is defined by the following form ${ }^{[9]}$.

$$
\boldsymbol{x}(s, t)=\frac{\sum_{i=1}^{n} \sum_{j=1}^{m} w_{i j} N_{i, p}(s) M_{j, p}(t) \boldsymbol{P}_{i j}}{\sum_{i=1}^{n} \sum_{j=1}^{m} w_{i j} N_{i, p}(s) M_{j, p}(t)}=\sum_{i=1}^{n} \sum_{j=1}^{m} R_{i, j}^{p, q}(s, t) \boldsymbol{P}_{i j} .
$$

Where, $\boldsymbol{P}_{i j}$ and $w_{i j}$ are surface's control points and weights seperately, $N_{i, p}(s)$ and $M_{j, p}(t)$ are B-spline basis function of degree $p$ and $q$, and $R_{i, j}^{p, q}$ is called as NURBS basis function. They are defined on their knot vectors $\boldsymbol{s}=\left[s_{0}, s_{1}, \ldots, s_{n+p}\right]$ and $\boldsymbol{t}=\left[t_{0}, t_{1}, \ldots, t_{m+q}\right]$, the definition of Bspline basis function is as following:

$$
\begin{gathered}
N_{i, 0}(s)=\left\{\begin{array}{cc}
0 & s \in\left[s_{i}, s_{i+1}\right) \\
0 & s \notin\left[s_{i}, s_{i+1}\right)
\end{array}\right. \\
N_{i, p}(s)=\frac{s-s_{i}}{s_{i+p}-s_{i}} N_{i, p-1}(s)+\frac{s_{i+p+1}-s}{s_{i+p+1}-s_{i+1}} N_{i+1, p-1}(s)
\end{gathered}
$$

The geometry's shape is described by assigning different control points. The knots can be refined through knot insertion algorithms, such as p-refinement, h-refinement. The former is realized by increasing the order of basis function and the latter is realized by increasing the number of basis function. However, compared with conventional FEM, the geometric shapes keep unchanged after refining.

\section{B. Isogeometric analysis}

The isogeometric analysis's basic concept is adopting the same basis function in the representation of geometry 
and physical field, so a more accurate geometry model is used in isogeometric analysis than traditional finite element method, so a more accurate solution can be obtained. According to the different of geometry representing method, there are isogeometric analysis methods based on B-spline, NURBS, or T-spline, of which isogeometric analysis based on NURBS is used in this study.

The basis function of $p$ and $q$ in NURBS surface have the continuity of up to $(p-1)$ and $(q-1)$ respectly. When more than two order basis functions are used, isogeometric analysis can keep the inter-element continuity of derivative field, hower, the conventional finite element method can only provide element-wise continuity.

In NURBS based isogeometric analysis, all the fields can be approximated as following.

$$
\boldsymbol{u}(s, t)=\sum_{i=1}^{n} \sum_{j=1}^{m} R_{i, j}^{p, q}(s, t) \boldsymbol{u}_{i j}
$$

Where $u_{i j}$ denotes the control variable of physical field such as displacement, strain and stress.

\section{LINEAR ELASTIC ANALYSIS AND STRESS CALCULATION}

Let $\Omega$ denotes the parametric domain bounded by $\Gamma$, where $\Gamma$ satisfies $\Gamma=\Gamma_{N} \cup \Gamma_{D}$ and $\Gamma_{N} \cap \Gamma_{D}=\varnothing . f$ is the body force. The elasticity problem on $\Omega$ can be defined as following.

$$
\left\{\begin{array}{lll}
\Delta \boldsymbol{u}=\boldsymbol{f} & \text { in } & \Omega \\
\boldsymbol{u}=\boldsymbol{g} & \text { on } & \Gamma_{D} \\
\frac{\partial \boldsymbol{u}}{\partial x} \boldsymbol{n}=\boldsymbol{t} & \text { on } & \Gamma_{N}
\end{array}\right.
$$

The second and third equations in (4) define Dirichlet and Neumann boundary condition separately, of which $\boldsymbol{g}$ and $\boldsymbol{t}$ denote the displacement on $\Gamma_{D}$ and the force on $\Gamma_{N}$. $\mathbf{n}$ is the unit outward normal vector on $\Gamma_{N}$. Formulation (4) is the strong form of elastic problem, but it can't be satisfied accurately. Applying Galerkin method, the strong form of the linear elastic problem can be approximated as its weak form with the following formulation.

$$
\int_{\Omega} \delta \varepsilon^{T} D \varepsilon d \Omega=\int_{\Gamma_{N}} t \boldsymbol{R} \delta \boldsymbol{u} d \Gamma+\int_{\Omega} \boldsymbol{R} f \delta \boldsymbol{u} d \Omega .
$$

Where $\boldsymbol{\varepsilon}$ is the strain and $\boldsymbol{D}$ is elastic matrix.

Considered $\boldsymbol{\varepsilon}=\boldsymbol{B} \boldsymbol{U}$, and (5) should be agreed with all $\delta \boldsymbol{u}$, the following formulation can be inferred.

$$
\int_{\Omega} B^{T} D B U d \Omega=\int_{\Gamma_{N}} t R d \Gamma+\int_{\Omega} R f d \Omega .
$$

Where $\mathbf{B}$ is strain matrix, and it is composed of the following submatrix:

$$
\boldsymbol{B}_{i}=\left[\begin{array}{cc}
\frac{\partial R_{i}(s, t)}{\partial x} & 0 \\
0 & \frac{\partial R_{i}(s, t)}{\partial y} \\
\frac{\partial R_{i}(s, t)}{\partial y} & \frac{\partial R_{i}(s, t)}{\partial x}
\end{array}\right]
$$

Let $\boldsymbol{K}=\int_{\Omega} \boldsymbol{B}^{T} \boldsymbol{D} \boldsymbol{B} d \Omega$ and $\boldsymbol{F}=\int_{\Gamma_{N}} \boldsymbol{t} \boldsymbol{R} d \Gamma+\int_{\Omega} \boldsymbol{R} f d \Omega$, we get the following equations:

$$
\boldsymbol{K} \boldsymbol{U}=\boldsymbol{F} .
$$

Since the integral in (6) can't be computed with analytic method, numerical integral has to be used to calculate stiffness matrix $\boldsymbol{K}$ and force matrix $\boldsymbol{F}$. Guass integral method is used in this study, and the detailed process can be referred to [8].

According to physics equation $\boldsymbol{\sigma}=\boldsymbol{D} \boldsymbol{\varepsilon}$ and the geometry equation, stress can be calculated as following:

$$
\sigma=\boldsymbol{D B U}
$$

\section{STRESS ACCURATE IMPROVED METHOD}

Strain and stress are high order differential vectors than displacement. Since the displacement solution may bring error in the weak form approximation and numerical integral, it may result in larger error after applying differential operator. During the process of numerical integral, the strain matrix and body forces at Gauss integral points are computed accurately, so the strain and stress calculating results are relatively more accurate than other positions. In order to take advantage of this phenomenon, two methods including stress interpolation method and least square fit method are given to improve the stress calcluate precision.

\section{A. Stress interpolation method}

Suppose the stress's distribution is the same as the displacement, it can be represented by NURBS basis function. The improved stress solution is expressed as following:

$$
\bar{\sigma}(s, t)=\sum R_{i, j}^{p, q}(s, t) \bar{\sigma}_{i j}
$$

Where $\bar{\sigma}_{i j}$ is the control variable of the improved stress solution.

The integral points in Guass integral are expressed as $\left[s_{k}, t_{l}\right], k=1, \ldots, n_{g 1}, l=1, \ldots, n_{g 2}$, the stresses at Guass integral points can be computed from the displacement solution by (7) and (8). Then we can build a corresponding equation on every Guass integral point according to (9). All the equations form the system of equations. 


$$
\bar{\sigma}\left(s_{k}, t_{l}\right)=\sum R_{i, j}^{p, q}\left(s_{k}, t_{l}\right) \bar{\sigma}_{i j} \quad k=1, \ldots, n_{g 1}, l=1, \ldots, n_{g 2} .
$$

Since the number of equations is greater than the unkowns, equations(10) belong to the overdetermined equations. They can not be satisfied exactly, so only the approximation solution may be obtained, which can be solved by least square method in matlab.

\section{B. Least square method}

In order to obtain the stress solution with the minimum error in the full domain, the weighted least square method can be used to get the approximation solution, which is realized by making the following formulation reach stationary value ${ }^{[8]}$.

$$
c(\bar{\sigma}, \sigma)=\int_{\Omega} \frac{1}{2}(\bar{\sigma}-\sigma)^{T} A(\bar{\sigma}-\sigma) d \Omega
$$

Here A stands for the weight factor matrix of least square method. Assumed that the improved stress solution is distributed as (9), put it into (11) and applying variation operator, the following equation can be gotten:

$$
\sum \frac{\partial c}{\partial \overline{\sigma_{i j}}} \delta \overline{\sigma_{i j}}=0
$$

Considered $\delta \overline{\sigma_{i j}}$ are arbitrary tiny values, the following systems of equation can be infered:

$$
\int_{\Omega}(\bar{\sigma}-\sigma)^{T} A R_{i, j}^{p, q} d \Omega=0 .
$$

If the equations' left sides are calculated with Guass integral method, and the weighted factor A is assigned to a unit matrix, the following systems of equation can be built.

$$
\sum_{k=1, \ldots, n_{g_{1}, l}, 1, \ldots, n_{g 2}}\left(\bar{\sigma}\left(s_{k}, t_{l}\right)-\sigma\left(s_{k}, t_{l}\right)\right)^{T} R_{i, j}^{p, q}\left(s_{k}, t_{l}\right) \varpi\left(s_{k}, t_{l}\right)=0 .
$$

Put (9) into (14), the equations with the unkowns of stress control variables can be obtained as following:

$$
\begin{aligned}
& \sum_{\substack{i=1, \ldots, n \\
j=1, \ldots, m}} \sum_{\substack{k=1, \ldots, n_{g_{1}} \\
l=1, \ldots, n_{g 2}}} R_{i_{0}, j_{0}}^{p, q}\left(s_{k}, t_{l}\right) R_{i, j}^{p, q}\left(s_{k}, t_{l}\right) \varpi\left(s_{k}, t_{l}\right) \bar{\sigma}_{i j} \\
= & \sum_{\substack{i=1, \ldots, n \\
j=1, \ldots, m}} \sum_{\substack{k=1, \ldots, n_{g_{1}} \\
l=1, \ldots, n_{g 2}}} R_{i_{0}, j_{0}}^{p, q}\left(s_{k}, t_{l}\right) \varpi\left(s_{k}, t_{l}\right) \bar{\sigma}\left(s_{k}, t_{l}\right) \quad i_{0}=1, \ldots, n, j_{0}=1, \ldots ., m .
\end{aligned}
$$

After solving the systems of equation, the stress control variables $\bar{\sigma}_{i j}$ can be used to calculate the stress's value at any position in the domain.

\section{THE PRACTICAL EXAMPLE}

The example in [7] about the infinite plate with circular hole under constant in-planc tension is used to verify the effect of stress improved calculation methods. Taking advantage of the symmetrical characteristic, only a finite quarter of the finite plate is analyzed to simulate the infinite plate problem. The infinite plate problem has the following exact solution ${ }^{[7]}$.

$$
\begin{aligned}
& \sigma_{r r}(r, \theta)=\frac{T_{x}}{2}\left(1-\frac{R^{2}}{r^{2}}\right)+\frac{T_{x}}{2}\left(1-4 \frac{R^{2}}{r^{2}}+3 \frac{R^{4}}{r^{4}}\right) \cos 2 \theta \\
& \sigma_{\theta \theta}(r, \theta)=\frac{T_{x}}{2}\left(1+\frac{R^{2}}{r^{2}}\right)-\frac{T_{x}}{2}\left(1+3 \frac{R^{4}}{r^{4}}\right) \cos 2 \theta \\
& \sigma_{r \theta}(r, \theta)=-\frac{T_{x}}{2}\left(1+2 \frac{R^{2}}{r^{2}}-3 \frac{R^{4}}{r^{4}}\right) \sin 2 \theta .
\end{aligned}
$$

Where $\sigma_{r r}, \sigma_{\theta \theta}, \sigma_{r \theta}$ are the stress in radial direction, tangential direction and shear stress in polar coordinate seperately. $T_{x}$ is the stress applied on the infinite plate along $x$ direction. If the stresses calculated by (16) are taken as Neumann boundary condition of the finite plate, the real stress state of infinite plate can be simulated. The length of the finite plate's edge is 4, and the hole's radius is 1 . Young's modulus and Poisson ratio of the plate is $0.21 \mathrm{Mpa}$ and 0.3 respectively. The coarse mesh is consisted of two elements, and its knot vectors are $[0,0,0,0.5,1,1,1],[0,0,0,1,1,1]$. Fig. 1a is the definition of the problem, and Fig. $1 \mathrm{~b}$ is the corresponding control net and element of the finite plate. Fig. $2 \mathrm{~b}$ is the stress computed result from the displacement after applying $h$-refinement on the initial mesh 3 times.

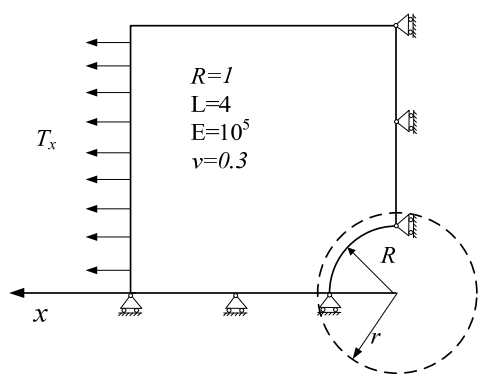

(a)

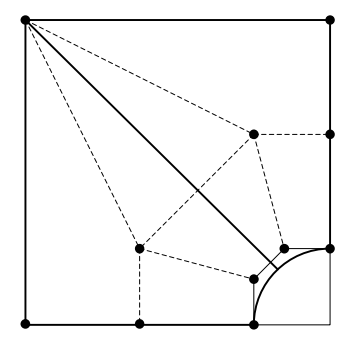

(b)
Figure 1 Plate with circular hole(a) Finite plate problem's definition (b) Initial control net

Fig. 2 is shown the origin computed results of $\sigma_{x}$ and $\sigma_{y}$ under the conditions that $T_{x}=10, p=q=3$ and the number of Guass integral method is 4 . Fig.3 gives the errors under different number of integral points and different refined times of h-refinement. Fig. 3a shows that the error of $\sigma_{x}$ decreases when the number of integral points increases from 2 to 10 after 1 time p-refinement, and the errors gotten by the two stress improved calculation methods are less than the error of original method when the integral points' number are more than 4 . The error of least square method is smaller than the stress interpolation method, but their differences are very small. When the number of integral points is more than 5, the error decrease unconspicuous.

Fig. 3b shows the errors of $\sigma_{x}$ decreases with the time of h-refinement when the order of NURBS is 3 and the integral 
points' number is 4 . The two improved methods reduce the stress calculate error, but their effects seem more and more slightly when the mesh's refinement time increases. The stress error of $\sigma_{x}$ used here is defined as follows:

$$
\delta_{x}=\sqrt{\sum\left(\sigma_{x}-\bar{\sigma}_{x}\right)^{2}}
$$

Where $\sigma_{x}$ is the exact analytic solution that can be calculated from $\sigma_{r r}, \sigma_{\theta \theta}$ and $\sigma_{r \theta}$, and $\bar{\sigma}_{x}$ is the improved stress result by our methods. The error of $\sigma_{y}$ and $\tau_{x y}$ can be disposed in a similar manner.

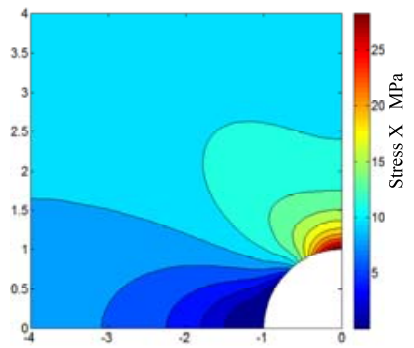

(a)

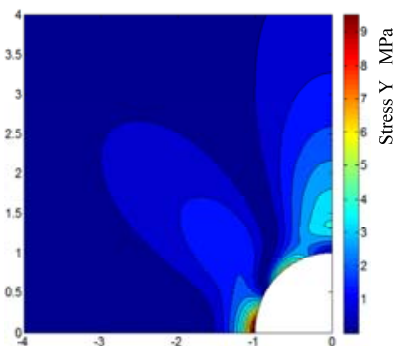

(b)
Figure 2 Stress analysis result by the improved calculated method ( $p=q=3, T_{x}=10,4$ integral points )(a) Stress $\mathrm{x}$ (b) Stress $\mathrm{y}$

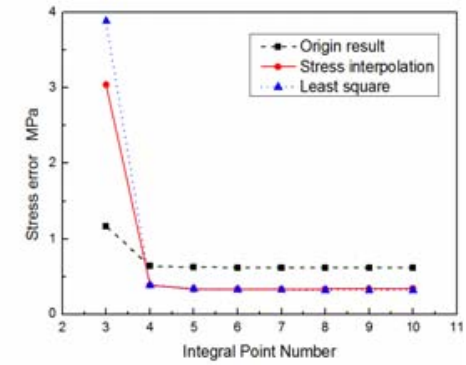

(a)

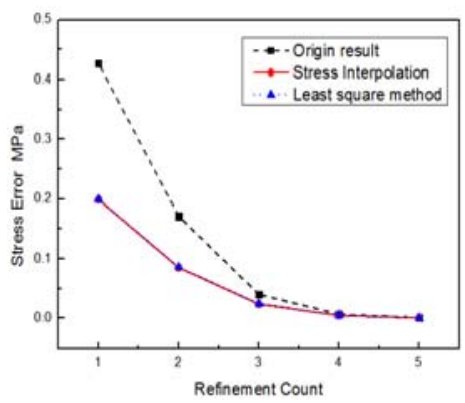

(b)

Figure 3 Stress analysis errors by the improved calculated methods

\section{CONCLUSION}

In order to change the fact that stress has relatively lower accuracy than displacement, two improve methods to calculate stress in isogeometric structure analysis. Based on the fact that stress calculated values in Guass integral point are more accurate than other locations, the concept that the stress field can be rebuilt by fitting the Guass integral point's stress from the displacement solution, and two methods including stress interpolation and least square fit are presented to improve the stress calculated result. The example of finite plate with circular hole is used to illustrate our methods' performance, and the results shows that our methods can improve the stress calculate accuracy notable. The error is decreased with the number of integral points, and the two proposed method have a similar accuracy. The following work includes using our methods into shape optimization and topology optimization process and other elastic problem.

\section{ACKNOWLEDGMENT}

This work was supported by the Fundamental Research Funds for the Central Universities. The authors would wish to thank the reviewers for their valuable comments.

\section{REFERENCES}

[1] Bazilevs Y., Calo V. M., Cottrell J. A., Evans J. A., et al. Isogeometric analysis using T-splines[J]. Computer Methods in Applied Mechanics and Engineering. 2010, 199(5-8): 229-263.

[2] Qian Xiaoping. Full analytical sensitivities in NURBS based isogeometric shape optimization[J]. Computer Methods in Applied Mechanics and Engineering. 2010, 199(29-32): 2059-2071.

[3] Seo Yu-Deok, Kim Hyun-Jung, Youn Sung-Kie. Shape optimization and its extension to topological design based on isogeometric analysis[J]. International Journal of Solids and Structures. 2010, 47(11-12): 1618-1640.

[4] Cottrell J. A., Reali A., Bazilevs Y., Hughes T. J. R. Isogeometric analysis of structural vibrations[J]. Computer Methods in Applied Mechanics and Engineering. 2006, 195(41-43): 5257-5296.

[5] Bazilevs Yuri, Zhang Yongjie, Calo Victor M., Goswami Samrat, et al. Isogeometric analysis of blood flow: A NURBS-based approach[C].Proceedings of the International Symposium CompIMAGE 2006 - Computational Modelling of Objects Represented in Images: Fundamentals, Methods and Applications Coimbra, Portugal: Taylor and Francis/Balkema, 2007.

[6] Temizer T., Wriggers P., Hughes T. J. R. Contact treatment in isogeometric analysis with NURBS[J]. Computer Methods in Applied Mechanics and Engineering. 2011, 200(9-12): 1100-1112.

[7] Hughes T. J. R., Cottrell J. A., Bazilevs Y. Isogeometric analysis: CAD, finite elements, NURBS, exact geometry and mesh refinement[J]. Computer Methods in Applied Mechanics and Engineering. 2005, 194(39-41): 4135-4195.

[8] Wang Xucheng. Finite element method[M]. Beijing: Tsinghua Univerisity Press, 2003.

[9] L. Piegl., Tiller W. The NURBS Book, Second ed.[M]. New York: Springer, 1997. 